\title{
Quantifying silo flow using MRI velocimetry for testing granular flow models
}

\author{
Luke Fullard* \\ School of Fundamental Sciences, \\ Massey University, New Zealand. \\ Daniel J. Holland \\ Department of Chemical and Process Engineering, \\ University of Canterbury, New Zealand. \\ Petrik Galvosas \\ School of Chemical and Physical Sciences, \\ Victoria University, New Zealand \\ Clive Davies \\ School of Food and Advanced Technology, \\ Massey University, New Zealand. \\ Pierre-Yves Lagrée, Stéphane Popinet \\ Institut Jean le Rond d'Alembert, \\ CNRS UMR 7190 \\ Sorbonne Université, \\ Paris, France. \\ ( Dated: September 10, 2020)
}

In this work we present experimental results of the gravity-driven discharge of poppy seeds from 3D-printed silos. The velocity fields of the flowing poppy seeds are measured using Magnetic Resonance Imaging (MRI) velocimetry techniques. Crucially, this approach allows the velocity field to be determined throughout the flow domain, unlike visual techniques such as Particle Image Velocimetry (PIV) and related methods where only the flow at or near the wall is accessible. We perform the experiment three times; with 3D-printed silos of cone half angles $30^{\circ}$ and $50^{\circ}$ respectively, and then repeat the $30^{\circ}$ silo experiment, but with a layer of poppy seeds glued to the silo wall to create a "rough wall" condition. In our experiments, we observe and quantify velocity fields for three well known granular flow regimes; mass flow, funnel flow, and rat-holing. The results of the experiments are compared to equivalent output of numerical simulations. In this mathematical model, the well-known $\mu(I)$ friction law is used to define an effective granular viscosity, and the flow is solved using a standard Navier-Stokes type solver. While the results are generally encouraging, it is noted that some aspects of the model are lacking and should be improved; in particular, the rat-holing effect observed in one of the MRI experiments was not predicted by the model, nor was the exact volumetric flow rate from any of the silos. Suggestions for model improvement are discussed.

\footnotetext{
* L.Fullard@Massey.ac.nz
}

\section{BACKGROUND AND INTRODUCTION}

Granular matter is well known to behave in complex and often unexpected ways. Particles in a granular assembly may act in a solid-like, liquid-like, or gas-like manner, with the transition between these phases often difficult to 
define and quantify [1]. A commonly studied 91 granular system is gravity-driven silo discharge. 92 In addition to being a system of great practical ${ }_{93}$ importance, silo flow can also display a vari- 94 ety of interesting flow dynamics. Depending on 95 the design of the silo (i.e. the silo half angle, 96 the friction between particles, the friction be- 97 tween the silo walls and particles, and the size ${ }_{98}$ and shape of the particles), the flow may be ei- ${ }_{99}$ ther mass-flow, funnel flow, or display rat-holing ${ }_{100}$ $[2,3]$. In mass flow, all particles in the silo are ${ }_{101}$ in motion with no stagnant zones; in funnel- ${ }_{102}$ flow there are regions within the silo where par- ${ }_{103}$ ticles flow, but there are also stagnant regions ${ }_{104}$ (and an interface between flowing/stagnant re- ${ }_{105}$ gions); when a silo displays rat-holing, flow only ${ }_{106}$ occurs in a central core approximately the size ${ }_{107}$ of the silo opening, with large stagnant regions ${ }_{108}$ surrounding this core. Rat-holing can be con- ${ }_{109}$ sidered an extreme case of funnel flow, but the flow is often observed to be intermittent and ${ }_{111}$ transient, whereas in a general funnel flow the $e_{112}$ dynamics are much more steady. Due to the ${ }_{113}$ variety of flow regimes, the silo provides an ex- ${ }_{114}$ cellent test of numerical models of granular dy- ${ }_{115}$ namics.

Apart from testing numerical codes, quantify-117 ing velocity fields in the silo is of great industrial ${ }_{118}$ importance, for example, in the study of parti-119 cle mixing and segregation as particle blends are 120 discharged from a silo. While there have been ${ }_{121}$ many Discrete Element Modelling (DEM) [4-122 6] and continuum models [7-14] developed to123 study the silo, experimental measurements and $\mathrm{d}_{124}$ validations are still required.

The vast majority of experimental character-126 isation of the velocity vector field in a discharg- ${ }^{127}$ ing silo has been using visual imaging methods ${ }^{128}$ in transparent silos (both conical and planar). ${ }^{129}$ Techniques such as Particle Image Velocime-130 try (PIV) and Particle Tracking Velocimetry ${ }^{131}$ (PTV) have been successfully applied to mea- ${ }^{132}$ sure the grain velocity at the silo walls [15-20]..133 On the contrary however, experimental mea-134 surements of velocity fields away from silo wall ${ }_{135}$ (i.e. in the bulk of the flow) are particularly $y_{136}$ difficult to obtain. Previous attempts to ex-137 perimentally quantify $3 \mathrm{D}$ velocity fields in silos 138 have included X-ray CT [21, 22], timing tracer discharge [23], Scanning gamma ray tomography $[24,25]$, and single profile proton absorptiometry [26], however, all of these methods give limited velocity profile information, and usually provide averaged data, data at discrete points, or data along a line only, rather than on a plane.

Magnetic Resonance Imaging (MRI) is an alternative technique that can study flow in optically opaque systems. MRI has been applied to non-silo granular systems [27-34] to quantify parameters such as velocity fields and packing. Kawaguchi [35] observed the flow type, either mass or funnel flow, in silos using tagged MR imaging. In this approach, bands of particles are tagged at one point in time and then the positions of these tagged particles imaged after a defined delay (in this case $100 \mathrm{~ms}$ ). The deformation of the tagged layers was observed visually. In theory this technique could be extended to estimate the velocity in a silo using further image processing techniques, but this would give only an indirect measure of the velocity fields. MRI has also been used to obtain the only reported direct, quantitative measurement of the silo velocity data on a plane away from the silo walls that we have found [36], though the range of silo flow conditions studied was limited. The first objective of the current article is to extend the work of Gentzler and Tardos [36] to obtain velocity field data for a wider range of silo flow situations. Firstly, we report on both the vertical and horizontal component of the velocity at the outlet. Secondly, we also measure particles of a large diameter $(\approx 1 \mathrm{~mm})$ such that the effect of the surrounding air on the particle dynamics near the orifice is not significant [37]. Thirdly, we consider the effect of changing the hopper geometry. Finally, we consider the effect of rough-walls on the particle dynamics. These last two aspects of the experiment mean that flow is studied across the three major flow regimes observed in silos.

A second objective is to assess the applicability of the so-called $\mu(I)$ friction law [38] for reproducing the velocity fields which we experimentally measure. Previously, the $\mu(I)$ friction law has been used to define an effective gran- 
ular viscosity for use in incompressible continuum flow models. Such an approach has been successfully applied to the granular column collapse and to some silo flows. [7, 8, 13, 39]. However, the velocity fields produced by the model have not been rigorously tested against experimental data. In particular, we examine the model applicability to reproduce the three silo flow modes, mass flow, funnel flow, and ratholing, which we observe in our experimental results.

\section{MATERIALS AND METHODS}

\section{Particle properties}

In this study, poppy seeds were chosen as the granular material of interest due to their particle size, their price and availability, and the fact that they contain abundant free oil which allows a strong signal to be detected by the MRI equipment. The poppy seeds were nonspherical, and were kidney shaped, as seen in Figure 1. The poppy long diameter was approximately $1.25 \mathrm{~mm}$, while the short diameter was approximately $0.85 \mathrm{~mm}$. A standard sieve experiment was performed and $\approx 93 \%$ of the particles were found to be between $710 \mu \mathrm{m}$ and $1180 \mu m$, with a Sauter mean diameter [40], $d$, of $951 \mu \mathrm{m}$.

\section{Silo system design}

The silo feeding system was designed to the specifications of the bore of the MRI apparatus in such a way that the poppy seeds were fully contained and never came in direct contact with the MRI apparatus itself. A system of perspex pipes of decreasing diameter was used to feed the poppy seeds into the test silo (the region to be imaged by the MRI) and then out of the bottom of the system. These pipes were con-181 nected using a series of push-fittings with small ${ }_{182}$ tolerances. Figure $2 \mathrm{~A}$. displays the full system ${ }_{183}$ of pipes and the test silo, while B. is a close 184 up of the silo itself. The silo was designed $i_{185}$
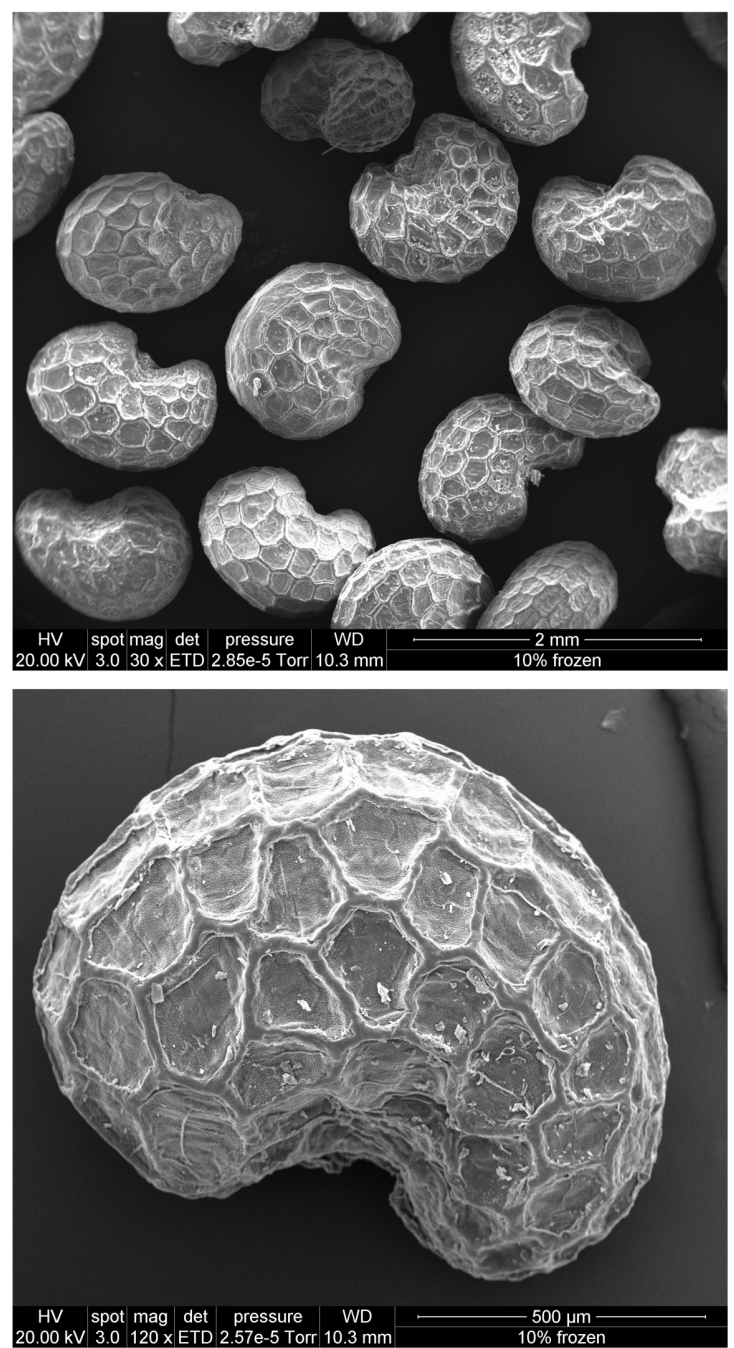

FIG. 1. Scanning Electron Microscope images of a sample of poppy seeds. It is apparent from the image that the seeds are non-spherical with a kidney shape. The surface of the seeds is also seen to be textured. A scale is included at the bottom of each image. A. An image of multiple poppy seeds. B. A close up of a single poppy seed.

a CAD program, 3D printed from ABS plastic, and the opening at the bottom of the silo, $D_{0}$, was drilled to a diameter of $6.5 \mathrm{~mm}$ (note that this is $\approx 6.5$ times greater than the Sauter mean diameter, $d$, of the particles to avoid jamming 
$[18,41])$. The inner diameter of the silo, $W$, was $23.5 \mathrm{~mm}$. Since $D_{0}>6.5 d, W>2.5 D_{0}$, and the bed height is always deeper than the silo opening diameter, the flow rate from the ${ }_{208}$ silo can be expected to be independent of the silo geometry. [42] The silo half angle, $\phi$, was changed between each experiment; the first silo ${ }^{209}$ had a $30^{\circ}$ half angle, the second $50^{\circ}$, and the ${ }^{210}$ third was another $30^{\circ}$ half angled silo but with ${ }^{211}$ rough walls. The rough walled silo was printed ${ }^{212}$ in two halves, then poppy seeds were glued onto ${ }^{213}$ the inner silo walls in a single layer, and finally, ${ }^{214}$ the two halves were glued together to form $\mathrm{a}^{215}$ full silo. We note that the diameter of the final ${ }^{216}$ pipe, labeled pipe 3 in Figure 2, was wider than ${ }^{217}$ the silo opening. This design was to avoid the ${ }^{218}$ well-known standpipe flow rate effect [43] which ${ }^{219}$ does not occur unless the pipe below the silo is ${ }^{220}$ full [43]. Since the silo opening diameter was $^{221}$ smaller than the exit pipe this was not the case ${ }_{223}^{222}$ and the standpipe effect was avoided.
A.

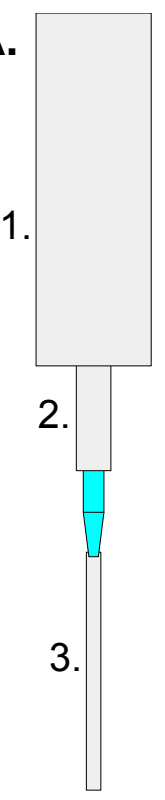

224

225 226

FIG. 2. A sketch of the piping and silo in the ex-246 perimental set-up (not to scale).

A. The 247 system is loaded from above. the seeds flow through $\mathrm{h}_{248}$ the largest pipe \#1. into the more narrow pipe \#2.249 through the test silo section, and out through pipe ${ }_{250}$

\section{Experimental method}

A Bruker Avance I Nuclear Magnetic Resonance spectrometer with a $9.4 \mathrm{~T}$ wide bore magnet located at Victoria University of Wellington, New Zealand was used for the experiments. A $30 \mathrm{~mm}$ diameter radio-frequency coil was used for excitation and detection. A three-axis shielded Micro2.5 gradient set capable of producing a maximum gradient strength of $1.51 \mathrm{~T}$ $m^{-1}$ was used for imaging and flow encoding. The pipes and silo were connected together and carefully inserted into the MRI. The silo and upper two pipes were filled from above through a funnel. A bucket was placed under the system to collect the discharged particles. As the particles were discharged the system was periodically refilled from above such that the upper pipe (pipe \#1) was never more than half empty. Note that the flow rate from the silo was constant and independent of fill height as is implicit in the Beverloo flow rate equation $[44,45]$.

The vertical (i.e. in the axial direction) and horizontal (i.e. in the radial direction) components of velocity of the poppy seeds were measured using a phase encoded velocity imaging sequence [46]. The image was obtained using a spin echo acquisition with a slice selective refocussing pulse. To enable accurate measurements of the wide range of velocities present in the system, experiments were repeated with 8 flow encoding gradients. The velocity was calculated from a linear fit to as many of these data points as possible. For the fastest flowing regions, typically only three experiments with the weakest flow encoding gradients were used, while in the slow moving regions all 8 experiments were used. The gradient encoding duration $\delta$ was set to $0.7 \mathrm{~ms}$, the observation time was $2.5 \mathrm{~ms}$, and the maximum gradient strength was set to $0.07 \mathrm{~T} \mathrm{~m}^{-1}$ in the vertical direction and $0.14 \mathrm{~T} \mathrm{~m}^{-1}$ in the horizontal direction. These settings gave a maximum field of flow of approximately $2 \mathrm{~m} \mathrm{~s}^{-1}$ with a minimum detectable velocity of $1 \times 10^{-3} \mathrm{~m} \mathrm{~s}^{-1}$, 
where the minimum detectable velocity corre-294 sponds to a signal-to-noise ratio for the phase 295 of 2. Images were acquired at a spatial reso-296 lution of $0.45 \mathrm{~mm}$ in the horizontal direction and $1.18 \mathrm{~mm}$ in the vertical direction with $\mathrm{a}_{297}$ slice thickness of $1 \mathrm{~mm}$. The total acquisition time for the images was approximately 50 minutes. Flow-encoded NMR images can acquire $\mathrm{a}^{298}$ phase arising from the imaging gradients themselves. It is common practice to correct this ${ }^{299}$ phase by acquiring measurements on a static sample. Here images of a static bed were also ${ }^{300}$ acquired. The phase change for these was negligible, thus no correction was required.

Three MRI experiments were performed, one with a silo of $30^{\circ}$ half angle, one with a silo of $50^{\circ}$ half angle, and finally with another silo ${ }_{303}$ of $30^{\circ}$ half angle, but with rough walls ( with $_{304}$ particles glued on the silo walls).

\section{Numerical model}

One goal of this work is to model the silo ${ }^{307}$ using a continuum model of granular flow. Re- ${ }^{308}$ cently, the $\mu(I)$ law for the friction of granular ${ }^{309}$ materials has been used to define an effective ${ }^{310}$ viscosity in granular flow simulations. This vis- ${ }^{311}$ cosity was successfully implemented into an in ${ }^{312}$ compressible Navier-Stokes solver (Gerris Flow ${ }^{313}$ Solver [47]) to model dense granular flow in $\mathrm{a}^{314}$ variety of situations $[7,8,13,39]$. For our sit- ${ }^{315}$ uation, an axisymmetric domain was used so ${ }^{316}$ that our 3D silo could be modelled in $2 \mathrm{D}$. The ${ }^{317}$ governing equations of incompressible flow were ${ }^{318}$ solved in Gerris;

$$
\begin{aligned}
\nabla \cdot \mathbf{u} & =0, \\
\rho\left(\frac{\partial \mathbf{u}}{\partial t}+\mathbf{u} \cdot \nabla \mathbf{u}\right) & =-\nabla p+\nabla \cdot(2 \eta \mathbf{D})+\rho \mathbf{g}(2)^{322}
\end{aligned}
$$

In the above continuity and momentum equa- ${ }_{325}{ }^{324}$ tions, $\mathbf{u}$ is the velocity vector, $\rho$ the flowing $_{326}$ (bulk) density, $p$ the local isotropic pressure, ${ }_{327}$ $\eta$ the effective (or apparent) granular viscosity ${ }_{328}$ and $\mathbf{D}$ the rate of strain tensor. The effective ${ }_{329}$ viscosity is defined as

$$
\eta_{e f f}=\frac{\mu(I) p}{D_{2}}
$$

but in practice a regularised effective viscosity was used to avoid infinite values when the fluid is experiencing small shear;

$$
\eta=\min \left(\frac{\mu(I) p}{D_{2}}, \eta_{\max }\right) \text {. }
$$

Here, $D_{2}=\sqrt{\frac{1}{2} D_{i j} D_{i j}}$ is the second invariant of the strain rate tensor, where $D_{i j}=\frac{\partial u_{i}}{\partial x_{j}}+$ $\frac{\partial u_{j}}{\partial x_{i}}$, and $\mu(I)$ is the granular friction law;

$$
\mu(I)=\mu_{1}+\frac{\mu_{2}-\mu_{1}}{I_{0} / I+1},
$$

with $\mu_{1}, \mu_{2}$, and $I_{0}$ parameters. The variable $I$ is the granular inertial number and is defined as

$$
I=\frac{d D_{2} \sqrt{\rho_{p}}}{\sqrt{p}},
$$

where $d$ is the particle diameter and $\rho_{p}$ is the solid particle density.

In our axisymmetric numerical model we apply no-slip conditions on both of the velocity components at the silo walls, a symmetry condition along the axis of symmetry, homogeneous Neumann velocity boundary conditions (for each velocity component) at the top and bottom of the silo, and we set $p=0$ at the top and bottom of the silo. Note that other boundary conditions could be used at the silo wall (for example, to allow slip at the silo wall $[48,49]$ ), but the effect of more complex boundary conditions is left for future work. For the $30^{\circ}$ silo with rough walls, the simulation domain was reduced by a particle diameter in size to account for the reduced dimensions due to the layer of particles glued to the silo walls, but the silo opening was kept at $6.5 \mathrm{~mm}$. No other change to the boundary conditions was made.

Parameters used in our simulation are listed in table I. The first friction parameter, $\mu_{1}$, was chosen based on measurements of the angle of repose of the poppy seeds which was found to be approximately $31^{\circ}$, hence, $\mu_{1}=\tan 31=0.6$. The upper limit on the friction angle, defined by parameter $\tan ^{-1}\left(\mu_{2}\right)$, was expected to be 
around $60^{\circ}$ since our MRI experimental results 368 for the velocity in the $30^{\circ}$ silo (to be presented 369 in Figure 3) showed small slow/stagnant regions370 at the transition from the conical to cylindri-371 cal section. We also noted that larger values372 of $I_{0}$ kept the incompressible $\mu(I)$ model in the well-posed regime for a wider range of inertial ${ }_{374}$ numbers than for low values of $I_{0}$ [50]. For this 375 reason, various values of $\tan ^{-1}\left(\mu_{2}\right) \approx 60^{\circ}$ and $_{376}$ $I_{0}$ between 0.05 and 1 were tested. It was found $\mathrm{d}_{377}$ that the parameters $\mu_{2}=1.7$ and $I_{0}=0.5$ gave 378 a good match to experimental data (to be dis-379 cussed), gave a wide range of well-posed inertial 380 number values, and, importantly, were physi-381 cally realistic.

TABLE I: Parameters used in the numerical model.

\begin{tabular}{|l|l|c|c|}
\hline \multicolumn{1}{|c|}{ Name } & Symbol & Unit & Value $_{386}^{385}$ \\
\hline Bulk density & $\rho$ & $\mathrm{kg} / \mathrm{m}^{3}$ & $600_{387}$ \\
\hline Particle density & $\rho_{p}$ & $\mathrm{~kg} / \mathrm{m}^{3}$ & $1_{380}$ \\
\hline Particle diameter & $d$ & $\mathrm{~mm}$ & $0.951_{3 \$ 9}$ \\
\hline Friction coefficient \#1 & $\mu_{1}$ & - & $0.6_{390}$ \\
\hline Friction coefficient \#2 & $\mu_{2}$ & - & 1.7 \\
\hline Reference inertial number & $I_{0}$ & - & 0.5
\end{tabular}

The results of the phase encoded velocity ${ }^{400}$ imaging sequence experiment were converted ${ }^{401}$ into a Matlab data file and plotted as a contour ${ }^{402}$ map. In Figure 3 the logarithm of the verti- ${ }^{403}$ cal component of velocity is plotted for each of the three silos, where $\mathbf{u}=(u, v)$ is the velocity ${ }^{404}$ vector with $u, v$ the horizontal and vertical velocity components respectively. The logarithm ${ }_{405}$ of the magnitude of the horizontal component 406 of velocity $(u)$ is shown in Figure 4. The lighter 407 (yellow) regions are zones of rapid flow, while 408 the darker (purple/blue) regions indicate slow 409 or stagnant flow. Horizontal velocity measure-410 ments were not available for the $30^{\circ}$ silo with ${ }_{411}$ rough walls because the magnitude of the hori-412 zontal component of velocity was very small and $\mathrm{d}_{43}$ was of the same order as the noise in the experiment.

The most immediate observation from Figure 3 is that for each silo we have a different flow regime. In the $30^{\circ}$ silo we observe mass flow. The particles in the silo at every location are in motion, with a possible small exception at the transition from the cone to the cylindrical section. In the $50^{\circ}$ silo we observe funnel flow. There is a region of flow in the center of the silo and this region of flowing material widens as we move further up into the silo. There is a clear stagnant region of flow that surrounds the flowing particles. This stagnant region shrinks as we transition higher into the silo. In the $30^{\circ}$ silo with rough walls (i.e. with a layer of poppy seeds glued to the wall) we observe the rat-holing effect. There is a fast core (roughly the diameter of the silo opening) of flowing particles surrounded by a region of stagnant material. The size of this stagnant zone does not perceptibly change as we transition higher into the silo. It is also apparent that the velocity field in the flowing zone remains continuous as we move higher in the silo, past the transition from the conical to cylindrical section (i.e. we do not observe velocity discontinuities or shocks). This is in contrast to predictions from Mohr-Coulomb plasticity based models $[2,51]$.

In order to assess the appropriateness of the incompressible assumption in our numerical model, we quantify the volumetric flow rate as a function of height above the silo opening. For each MRI experiment we use the vertical component of velocity $(v)$ to calculate the volumetric flow rate;

$$
Q(z)=2 \pi \int_{r(z)=0}^{r(z)=R(z)} v r d r,
$$

where $r(z)$ is the radial coordinate from the axis of the silo, and $R(z)$ is the radius of the silo at height $z$ above the opening. The resulting flow rates for each experiment are plotted in Figure 5.

It is apparent from the figure that the volumetric flow rate is approximately constant throughout the silo in the $30^{\circ}$ silo, but this is not so for the $50^{\circ}$ and $30^{\circ}$ silo with roughened 
Vertical velocity. $30^{\circ}$ silo

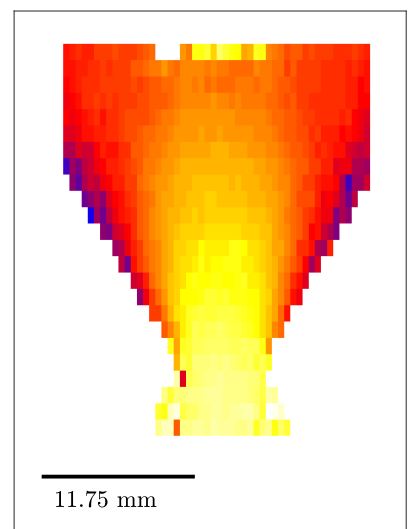

$30^{\circ}$ silo, rough wall

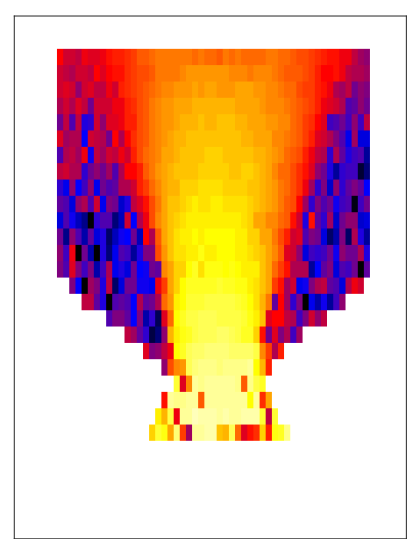

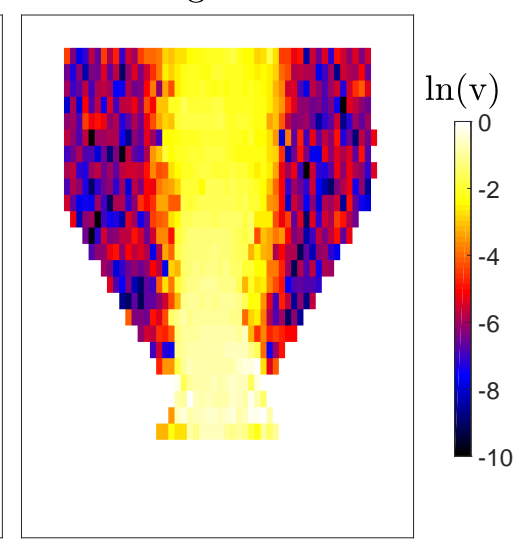

FIG. 3. The log of the magnitude of the vertical component of velocity $(v)$ is plotted for each of the three silos. Mass flow is observed in the $30^{\circ}$ silo, funnel flow in the $50^{\circ}$, and rat-holing in the $30^{\circ}$ silo with rough walls (with particles glued to the silo wall). Yellow regions indicate rapid flow, while purple/blue areas indicate slow to stagnant zones.

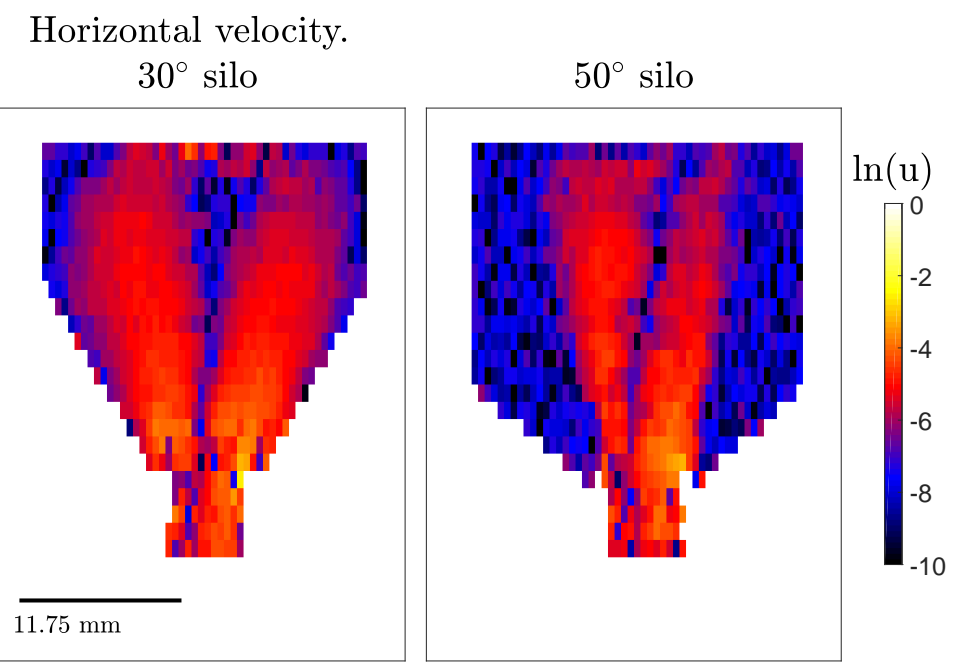

FIG. 4. The log of the magnitude of the horizontal component of velocity $(u)$ for the $30^{\circ}$ and $50^{\circ}$ silos.

walls. In these two non-constant flow rate cases, 420 the volumetric flow rate $Q(z)$ is seen to be $\approx 2 \times_{421}$ higher near the opening than it is in the bulk of ${ }_{422}$ the silo. This variation in flow rate could arise 423 either from a measurement error or a dilation ${ }_{424}$ of the flow at the outlet. The signal intensity ${ }_{425}$ at the outlet in all three images is less than half that in the bulk, which would be consistent with a dilation of the flow at the outlet. However, in these measurements there is also significant attenuation of the signal due to the motion of the particles, so the images are not quantitative 


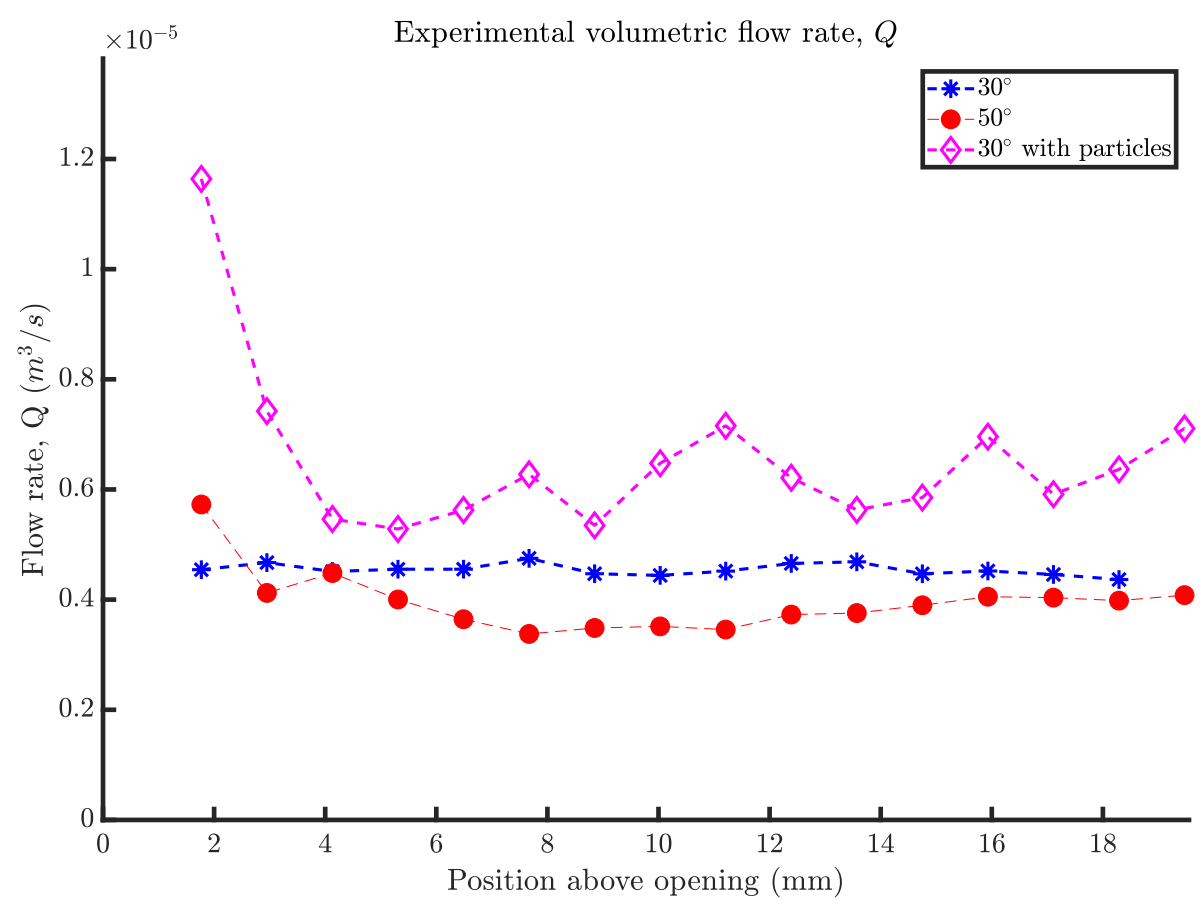

FIG. 5. The volumetric flow rate, $Q(z)$, for each of the three silo experiments as a function of height above the silo opening.

in solid fraction. Therefore it is important to 447 consider the errors that arise in measurement 448 of velocity. MRI measurements of the velocity 449 are prone to error in regions of high velocity,450 but this error will tend to cause an underesti-451 mation of the velocity as faster moving particles 452 are more heavily attenuated than slower moving $_{453}$ particles. The flow rate is seen to increase to-454 wards the outlet, hence, it is unlikely that a ve-455 locity measurement error could explain the ob-456 served flow rate variation. Therefore, it is con-457 cluded that, for the funnel flow and rat-holing ${ }_{458}$ silos, there is significant dilation of the flow near 459 the opening, and the assumption of incompress-460 ibility is likely to be erroneous, at least near ${ }_{461}$ the silo opening. In a similar system, a wedge ${ }_{462}$ shaped hopper, a significant reduction in bulk ${ }_{463}$ density has been observed [52]. As a point of ${ }_{464}$ context, in the numerical model the incompress-465 ibility condition is enforced (up to a tolerance) ${ }_{466}$ and it was found that the change in the volumet-467 ric flow rate was less than $1 \%$ throughout the silo. Here we assume that the use of an incompressible flow model has only a small effect on the predicted velocity fields, since in the bulk of the silo the flow rate is relatively constant, changing only near the silo opening. However, the dilation near the opening will change the predicted flow rate values. Given this result and model assumption, when comparing experimental and numerical results with an incompressible flow assumption, the velocity should be adjusted to account for the change in volumetric flow rate. In practice this is achieved by normalising the velocity by the volumetric flow rate at each local height above the silo opening. Furthermore, we quantified the mass flow rate, $\dot{m}$, from each of the silos by measuring the mass ejected from the system in a given time. For the $30^{\circ}$ silo we found $\dot{m}_{30}=2.11 \pm 0.07$ $\mathrm{g} / \mathrm{s}$, for the $50^{\circ}$ silo, $\dot{m}_{50}=1.74 \pm 0.09 \mathrm{~g} / \mathrm{s}$, and for the $30^{\circ}$ silo with particles on the wall, 
$\dot{m}_{30}^{p}=2.2 \pm 0.1 \mathrm{~g} / \mathrm{s}$. The reduction of the $\mathrm{S}_{512}$ mass flow rate between the $30^{\circ}$ and $50^{\circ}$ silos 513 is compared with corrections made to the Bev-514 erloo flow rate to account for hopper half angle 515 [53]. Assuming that the Beverloo parameters516 and bulk density is equal between the two silos ${ }_{517}$ of differing half angles, the ratio of the two flow ${ }_{518}$ rates is given as $M=\frac{f\left(50^{\circ}\right)}{f\left(30^{\circ}\right)}$, where the func-519 tion $f(\alpha)=\sqrt{\frac{1-\cos \alpha}{2 \sin ^{3} \alpha}}$. The theoretical ratio $M_{521}^{520}$ is calculated as 0.86 , while the experimental ra-522 tio in our system, $\frac{\dot{m}_{50}}{\dot{m}_{30}}$ is found to be $0.82 \pm 0.05,523$ in good agreement with the theoretical value. ${ }_{524}$

To directly compare the $\mu(I)$ numerical re--529 sults to the MRI experimental results a results ${ }^{530}$ file was imported from Gerris into Matlab which ${ }^{53}$ contained vertical and horizontal components of ${ }^{532}$ velocity. This data was interpolated onto five ${ }^{533}$ horizontal lines which correspond to the loca-534 tions of measurements taken in the MRI experiments. Thus, the horizontal and vertical components of velocity predicted in the model could ${ }^{535}$ be directly compared to the experimental data.

As previously mentioned, the volumetric flow 536 rate in the silo experiments was not a constant537 near the opening of the silo. Therefore, both $h_{538}$ the experimentally measured and numerically ys9 $_{53}$ predicted velocity data were normalised by the ${ }_{540}$ volumetric flow rate before being compared. At ${ }_{541}$ each height above the silo opening, $z$, the local ${ }_{542}$ volumetric flow rate is calculated using Equa-543 tion 7 . The velocity components are then mul-544 tiplied by the particle diameter squared and di-545 vided by the local volumetric flow rate to obtain ${ }_{546}$ the normalised velocity, $\widetilde{\mathbf{u}}$, where $\widetilde{\mathbf{u}}=\mathbf{u} d^{2} / Q .{ }_{547}$

The comparison of the vertical velocity pro-548 file taken at five heights above the opening for 549 the $30^{\circ}$ silo with smooth walls (i.e. no parti-550 cles attached to the wall) is shown in Figure 6,551 while the horizontal velocity profile is shown in 552 Figure 7 . The distance from the silo opening 553 to the silo transition (the point where the cone 554 becomes a cylinder) is $\approx 14.7 \mathrm{~mm}$, hence four 555 of the comparison lines are in the converging 556 conical section of the silo, while one is in the cylindrical section.

It is apparent that the match between the experimentally derived and numerically predicted normalised velocity is good, particularly for the vertical velocity. The normalised velocity predicted by the model has approximately the same maximum and also approximately the same curvature and shape as the MRI experimental measurements. However, the absolute velocity predicted by the model does not match the experiment due to the discrepancy in the volumetric flow rate between the two. There is more noise in the horizontal measurements, and the prediction of normalised horizontal velocity is slightly worse near the silo opening, but overall the agreement is satisfying.

As a further test, in Figure 8 we plot the normalised vertical component of velocity along the axial centerline of the silo and compare the experiment to the model. It is apparent that the model prediction is in very good agreement with the experimental results.

\section{Numerical Model Results: $50^{\circ}$ silo}

In Section III 2, the comparison of numerical and experimental velocity fields for the $30^{\circ}$ silo with smooth walls, there were no stagnant regions in the flow domain. The transition from flowing to stationary is difficult to capture with simple incompressible Navier-Stokes based models. Figures 9 and 10 show the normalised vertical and horizontal velocity measurements and predictions in the $50^{\circ}$ silo. In this silo the distance from the silo opening to the transition point is $\approx 7.1 \mathrm{~mm}$, hence in this case two of our velocity contours are in the conical section, while the remaining three are in the cylindrical section.

Remarkably, the match between experimental and numerical model results is quite good. Despite the observed transition from a flowing to a stagnant state in the silo domain, the granular viscosity model is able to capture the (normalised) maximum velocity, the curvature and shape of the velocity contours, and the approx- 


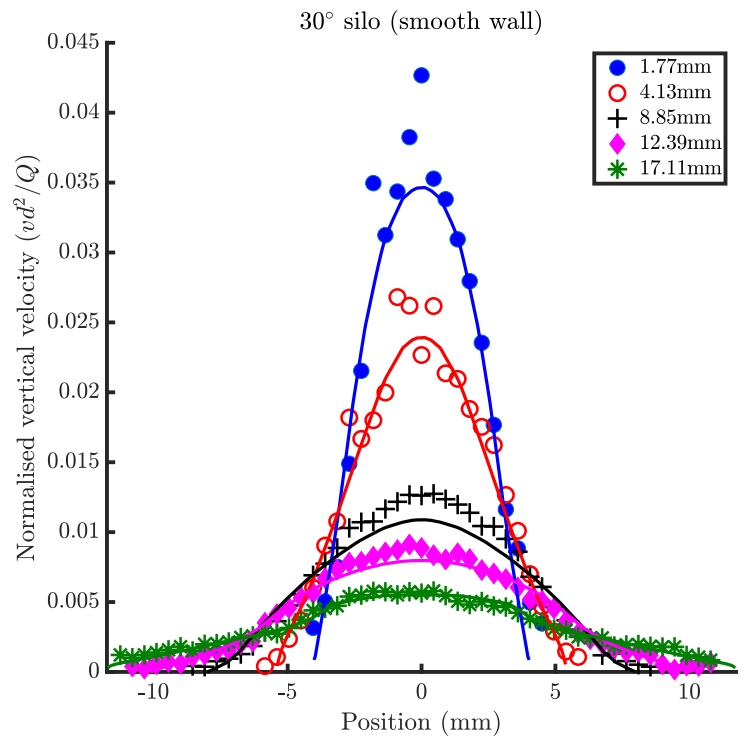

FIG. 6. The vertical velocity MRI measurements (solid circles) compared with those predicted by the numerical model (lines) for the $30^{\circ}$ silo.

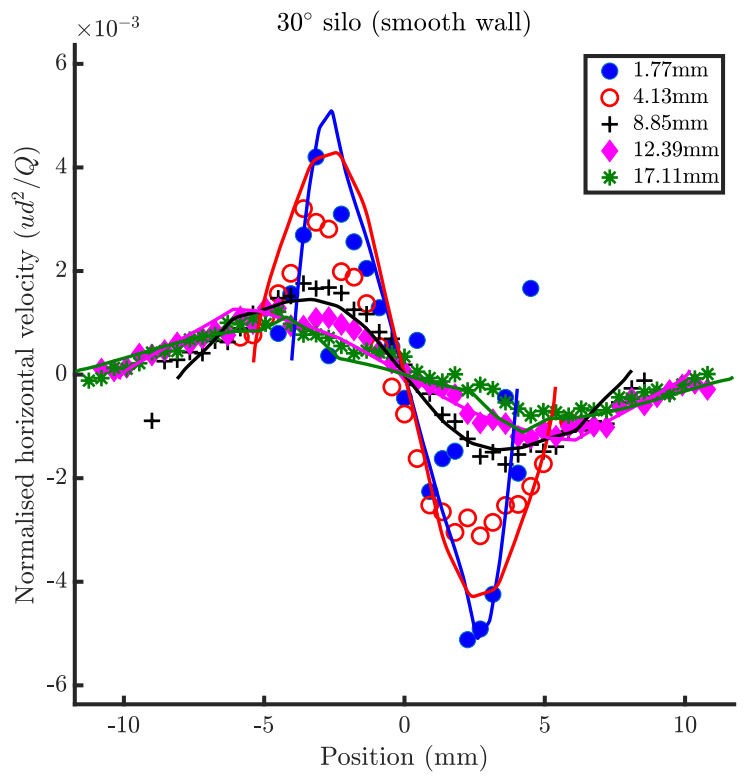

FIG. 7. The horizontal velocity MRI measurements (solid circles) compared with those predicted by the numerical model (lines) for the $30^{\circ}$ silo. at the same locations as in the vertical velocity figure. 


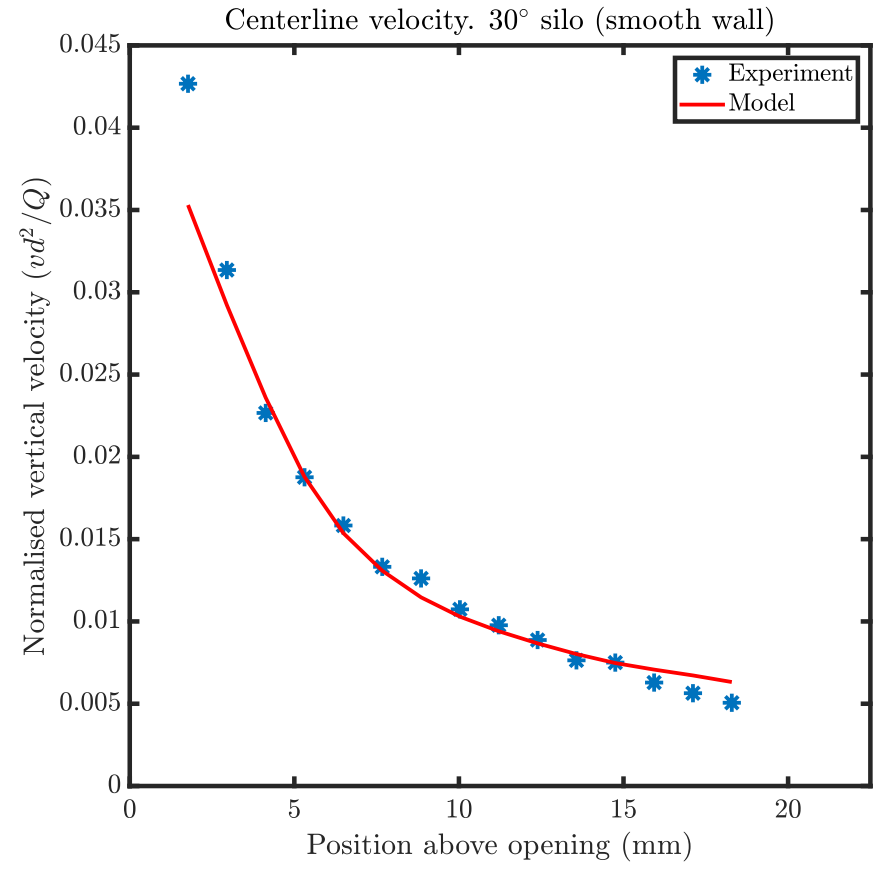

FIG. 8. A comparison of the normalised vertical velocity measured along the axial centerline of the silo compared with that predicted by the model for the $30^{\circ}$ silo. 


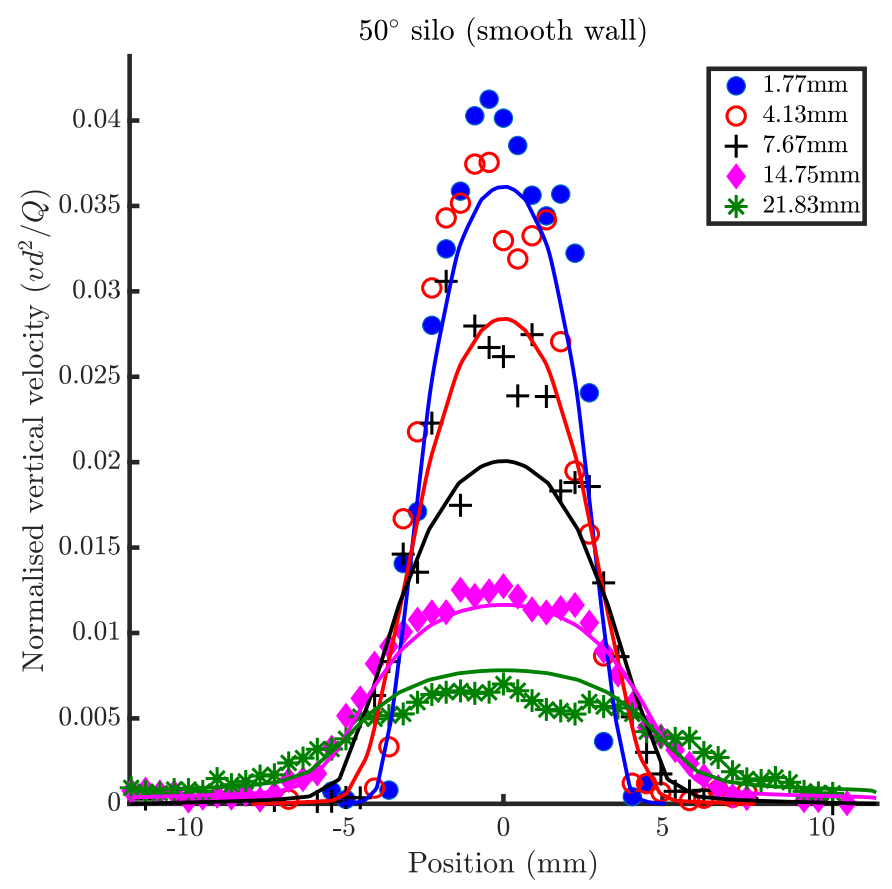

FIG. 9. The vertical velocity MRI measurements (solid circles) compared with those predicted by the numerical model (lines) for the $50^{\circ}$ silo.

imate location of the solid/flowing boundary. 575

Figure 11 compares the model to experimen-576 tal normalised vertical velocity along the axial577 centerline of the $50^{\circ}$ silo. In this case the ex-578 perimentally measured velocity contains more579 noise than in the $30^{\circ}$ case, but it is apparent580 that the model and experiment are of similars81 and follow a somewhat similar decrease. How-582 ever, the comparison is not quite as good as in ns3 $_{53}$ the $30^{\circ}$ case.

\section{Numerical Model Results: $30^{\circ}$ silo with rough} walls

The most challenging flow regime to replicate ${ }^{588}$ is the rat-holing behaviour observed in the $30^{\circ}$ silo with roughened walls. In this case the ob-589 served magnitudes of horizontal velocity were 590 too small to quantify since they were impercep-591 tible from the experimental noise. Hence, the 592 comparison of experimental to numerical predictions was only possible for the vertical velocity component. Figure 12 displays the normalised vertical velocity profile at five heights above the silo opening, while Fig. 13 is the normalised vertical velocity measured and predicted along the axial centerline of the silo.

It is apparent that the $\mu(I)$ model predictions completely fail to replicate the measured velocity, particularly far from the silo opening. In the case of rat-holing flow, the $\mu(I)$ model is unable to capture the observed dynamics.

\section{Numerical Model: Sensitivity analysis and flow rates}

In order to further compare the experimental and numerical velocity predictions we compare predicted flow rates between the numerical and experimental results, and perform a sensitivity 


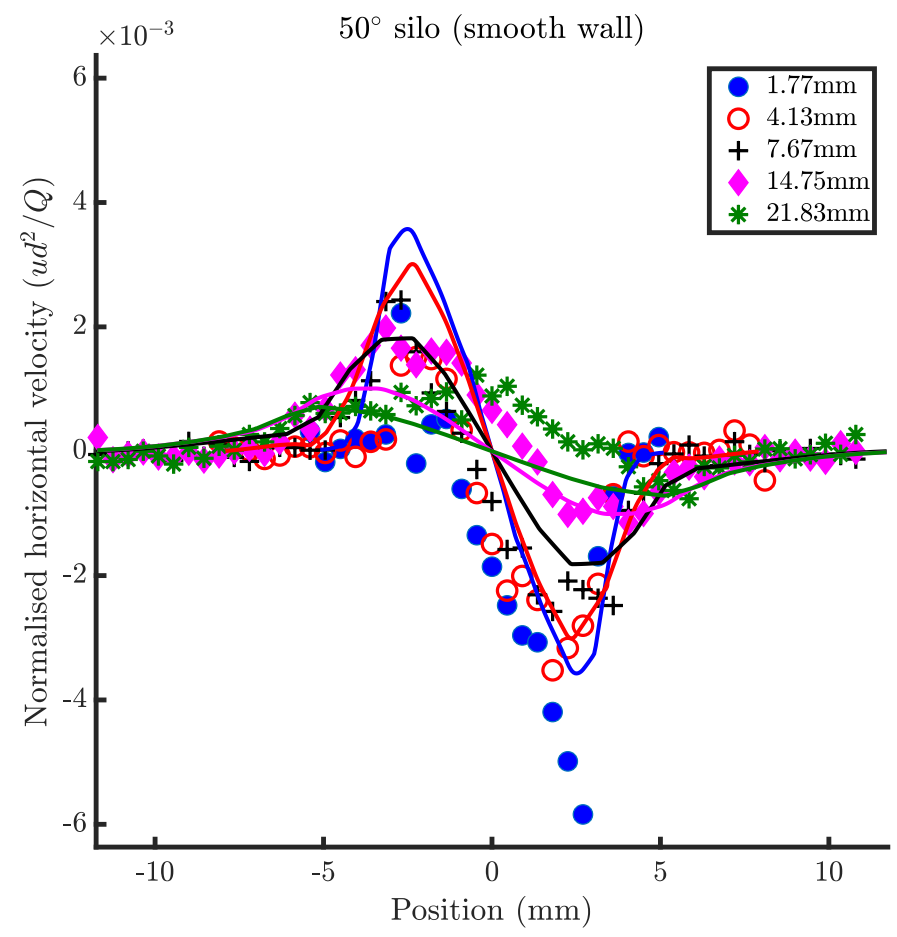

FIG. 10. The horizontal velocity MRI measurements (solid circles) compared with those predicted by the numerical model (lines) for the $50^{\circ}$ silo. at the same locations as in the vertical velocity figure.

analysis on the numerical model parameters. $\quad{ }_{613}$

To quantify the "goodness of fit" of the nu- ${ }^{618}$ merical predictions of velocity to the experi- ${ }^{-616}$ mentally measured ones we perform linear least ${ }^{-617}$ squares regression on the normalised vertical ve ${ }^{618}$ locity data: $\widetilde{v}_{\text {num }}=b \widetilde{v}_{\text {exp }}$ (i.e. we force the re $^{-{ }^{619}}$ gression to pass through the origin). In the $\operatorname{case}^{620}$ of a perfect fit between the numerical and $\mathrm{ex}^{-{ }^{621}}$ perimental data, the slope of the line, $b$, would ${ }^{622}$ be unity. The normalised vertical velocity data ${ }^{623}$ at five heights above the silo opening (the same ${ }^{624}$ heights as used in Figures 6,9) are combined $^{625}$ and the regression is performed on the entirety ${ }^{626}$ of this data at once. To test the sensitivity ${ }^{227}$ of the model predictions to model parameters ${ }^{628}$ this process was repeated 65 times for differ $^{629}$ ent values of $I_{0}$ and $\mu_{2}$. This analysis was per- ${ }^{630}$ formed for both the $30^{\circ}$ and $50^{\circ}$ silos, resulting ${ }^{631}$ in 130 numerical simulations. In each simula ${ }^{632}$ tion the value of $\mu_{1}$ was kept constant at $0.6{ }^{633}$ while the ranges of the other two parameters were $0.05<I_{0}<1$, and $0.9<\mu_{2}<2.1$. In Figure 14 the slopes resulting from the linear leastsquares regression analysis are contoured for the $30^{\circ}$ (left) and $50^{\circ}$ (right) silo flows respectively. The solid red dot in the contour plots indicates the values of the parameters used in the current work to produce Figures 6 - 13. The fine red line in the left plot is the contour of slope $=1$ which represents a perfect fit of the numerical prediction of normalised vertical velocity to its experimental measurement. In general, the $30^{\circ}$ silo numerical simulation was better fit for lower $I_{0}$ and larger $\mu_{2}-\mu_{1}$ values, while the $50^{\circ}$ simulation had the opposite behaviour. The $30^{\circ}$ simulation was always better fit to the experimental data than the $50^{\circ}$ one, with reported slopes in the range 0.86 to 1.03 (by comparison, the $50^{\circ}$ silo slopes were in the range 0.65 to 0.89 ). For the parameters used in the main 


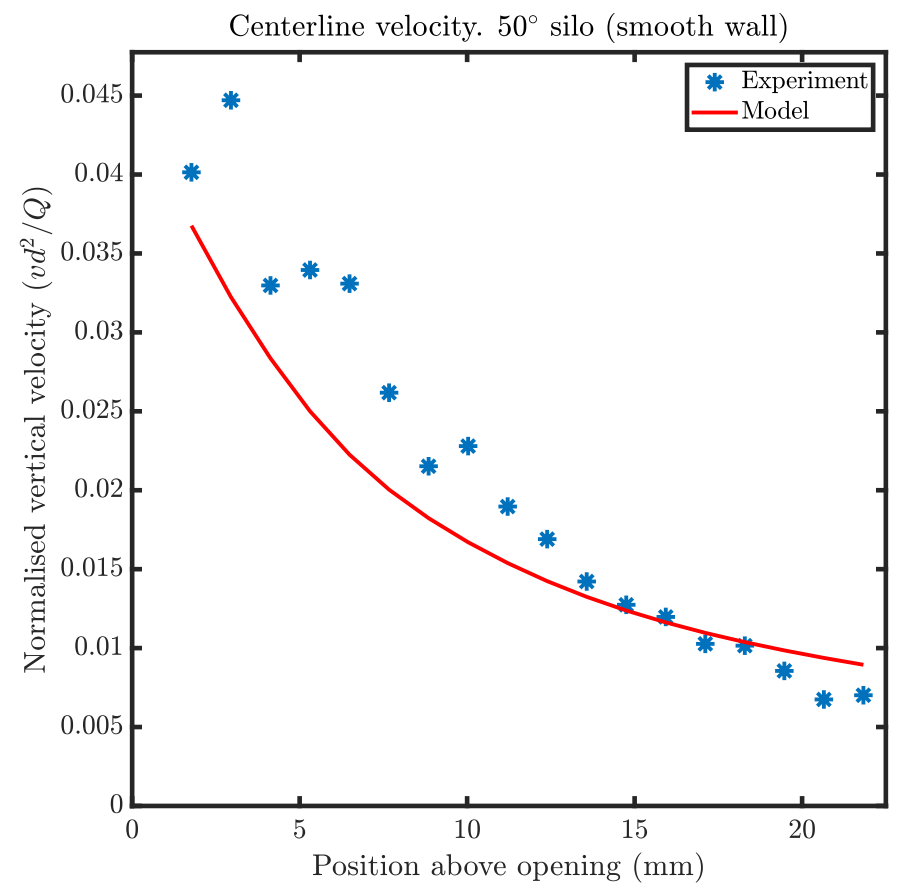

FIG. 11. A comparison of the normalised vertical velocity measured along the axial centerline of the silo compared with that predicted by the model for the $50^{\circ}$ silo.

text (see Table I) the least squares slopes were 655 0.94 for the $30^{\circ}$ silo, and 0.84 for the $50^{\circ}$ one. .56 Overall, the choice of the parameters $I_{0}=0.5657$ and $\mu_{2}=1.7$ used in this work is shown to be658 a good balance between accuracy for both the659 $30^{\circ}$ and $50^{\circ}$ silos.

Table II presents, for each of the three silos, ${ }_{662}{ }_{662}$ the experimentally derived mass and volumetric ${ }_{663}$ flow rates, the numerically predicted volumet- ${ }^{663}$ ric flow rate, and an approximate solids volume fraction in the bulk of the silo. The solids vol ${ }_{666}^{665}$ ume fraction in the bulk was approximated by ${ }_{667}^{660}$ taking the ratio of the experimental mass and ${ }_{668}^{667}$ volumetric flow rates (in the bulk of the silo), then dividing by the particle density $(\approx 1000$ $\mathrm{kg} / \mathrm{m}^{3}$ ). The predicted flowing solids fraction in the bulk of the $30^{\circ}$ and $50^{\circ}$ silos is remark- ${ }^{669}$ ably similar (0.46 and 0.47 respectively). However, the $30^{\circ}$ silo with particles glued to the wall ${ }_{670}$ shows a significantly lower solids volume frac-671 tion of 0.36 . As previously noted, the numer-672 ical model was of incompressible type, hence was not able to accurately predict the correct flow rate. In the table the predicted volumetric flow rate in the $30^{\circ}$ silo simulation was a factor of $\approx 4.5$ smaller than the experimentally observed one. The volumetric flow rate predicted in the $50^{\circ}$ silo simulation was a lot closer to the experimentally observed rate, but we caution against interpreting this as a validation of the model. During the sensitivity analysis the predicted flow rate varied by a factor of ten over the ranges of the parameters tested, which indicates that it is sensitive to model parameter choice.

\section{DISCUSSION AND CONCLUSIONS}

In this work we have presented results of experimental and numerical investigation of silo flow in three flow regimes; mass flow, funnel 


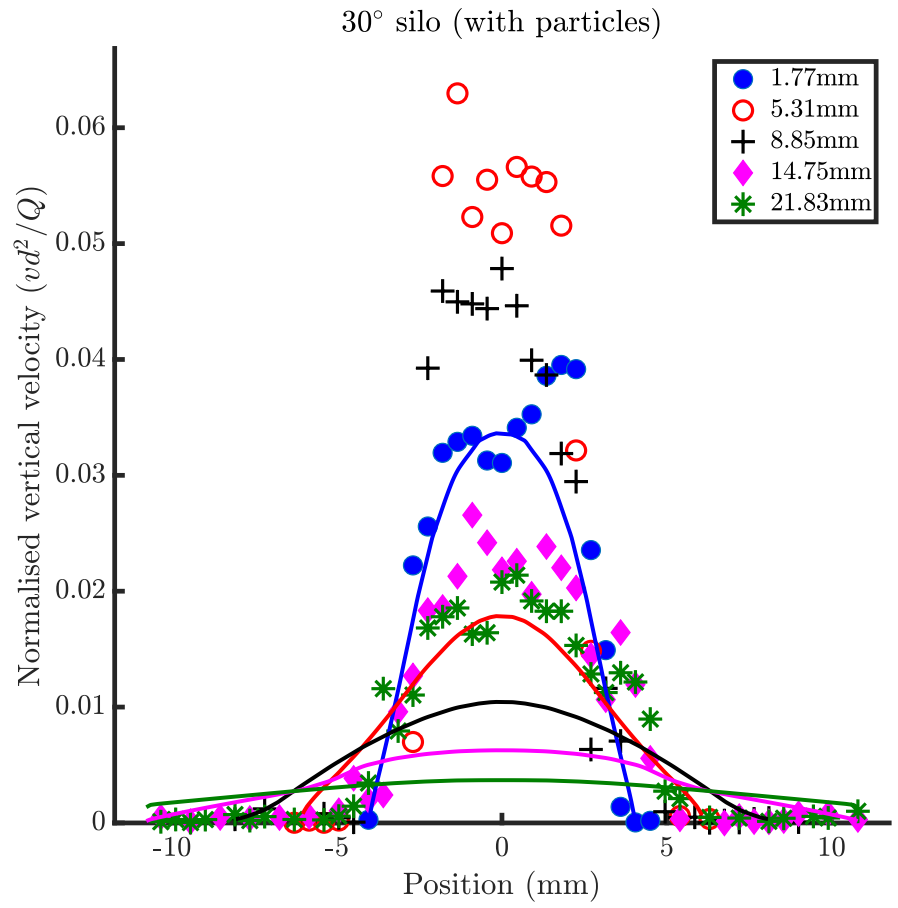

FIG. 12. The vertical velocity MRI measurements (solid circles) compared with those predicted by the numerical model (lines) for the $30^{\circ}$ silo with roughened walls.

TABLE II: The experimentally derived and numerically predicted flow rates in the tested silos.

\begin{tabular}{|c|c|c|c|c|}
\cline { 2 - 5 } \multicolumn{1}{c|}{} & $\dot{m}(\mathrm{~g} / \mathrm{s})$ & $Q_{\exp }\left(\mathrm{bulk}, \mathrm{cm}^{3} / \mathrm{s}\right)$ & $Q_{\text {num }}\left(\mathrm{cm}^{3} / \mathrm{s}\right)$ & $\sim \phi_{\exp }=\left(\dot{m} / Q_{\exp }\right) / \rho_{p}$ \\
\hline $30^{\circ}$ & $2.11 \pm 0.07$ & $4.54 \pm 0.05$ & 0.97 & $0.47 \pm 0.02$ \\
\hline $50^{\circ}$ & $1.74 \pm 0.09$ & $3.8 \pm 0.1$ & 4.2 & $0.46 \pm 0.04$ \\
\hline $30^{\circ}$ (with particles) & $2.2 \pm 0.1$ & $6.1 \pm 0.3$ & 0.81 & $0.36 \pm 0.04$ \\
\hline
\end{tabular}

flow, and rat-holing. Using MRI velocimetry we687 measured both the horizontal and vertical com-688 ponents of velocity throughout the three test689 silos, including the transition from the converg-690 ing conical to the cylindrical section. We found691 that the $30^{\circ}$ silo produced a mass flow, the $50^{\circ}{ }_{692}$ silo produced a funnel flow, and the $30^{\circ}$ silo with693 rough walls produced a rat-holing flow. We also694 presented results of a numerical model which695 used the $\mu(I)$ friction law to define an effec-696 tive granular viscosity for dense granular flow.697 This viscosity was used to simulate the silo flows698 by means of incompressible computational fluid 699 dynamics.
It was observed that the apparent volumetric flow rate in the MRI experiments was constant in the $30^{\circ}$ silo, but was a function of height above the silo opening for the other two; the flow rate was large near the silo opening but then rapidly fell to a near constant higher in the silo. The flow rate near the opening was roughly $2 \times$ that of the bulk, indicating that there is significant dilation of the flow near the silo exit opening in the $50^{\circ}$ and $30^{\circ}$ with rough wall cases. This is in contrast to the numerical model which enforced incompressibility of the flow. Recent studies have quantified the effect of solids fraction value at the silo opening on the 


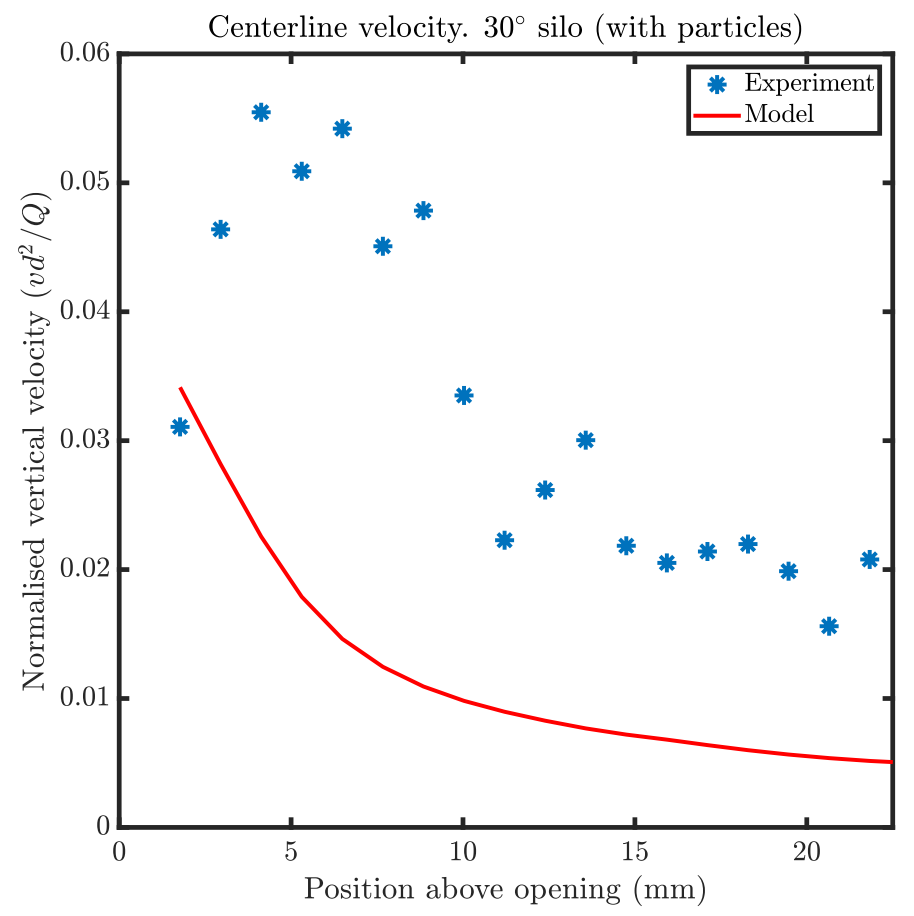

FIG. 13. A comparison of the normalised vertical velocity measured along the axial centerline of the silo compared with that predicted by the model for the $30^{\circ}$ silo with roughened walls.

flow rate from the silo [54], and reported that 721 solids fraction in the near opening region could ${ }_{722}$ be as low as half that in the bulk of the silo. Weт23 conclude that to fully capture the experimental ${ }_{724}$ measurement of the flow rate (and hence, the 725 exact values of velocity) numerical models will 726 likely need to include dilation effects, particu-727 larly for funnel and rat-holing flows. The ef-728 fect of dilation for the mass flow silo appeared ${ }_{729}$ negligible, but may be important to accurately 730 predict the volumetric flow rate from the silo. 731

To allow comparison between our experimen- ${ }_{733}^{732}$ tal and numerical results, the velocity compo- ${ }_{734}$ nents of each were normalised by the local value of volumetric flow rate (i.e. the flow rate at 735 height $z$ above the silo opening). The resulting ${ }_{736}$ velocity fields derived from the $30^{\circ}$ silo simu-737 lation showed excellent agreement with the ex-7зs perimental data. Plots of the vertical and hor-739 izontal velocity at a series of heights above the $e_{740}$ opening showed that both the shape and (normalised) maximum of the velocity contours were well matched, as was the vertical velocity component measured along the center-line of the silo. The comparison in the $50^{\circ}$ silo (which operated in the funnel flow regime in the MRI experiment) were surprisingly impressive, with very good agreement between experimental and numerical results. This suggests that for appropriate values of fitting parameters the $\mu(I)$ friction law can be used to define an effective granular viscosity for granular dynamics, even in the case where there are transitions from static to flowing regions in the domain of study.

However, for the $30^{\circ}$ silo with roughened walls (which displayed rat-holing in the MRI experiment), the simulation results were poorly matched to the experimental data. The grain dynamics in this silo are very complicated and hard to capture with numerical models. Rat- 

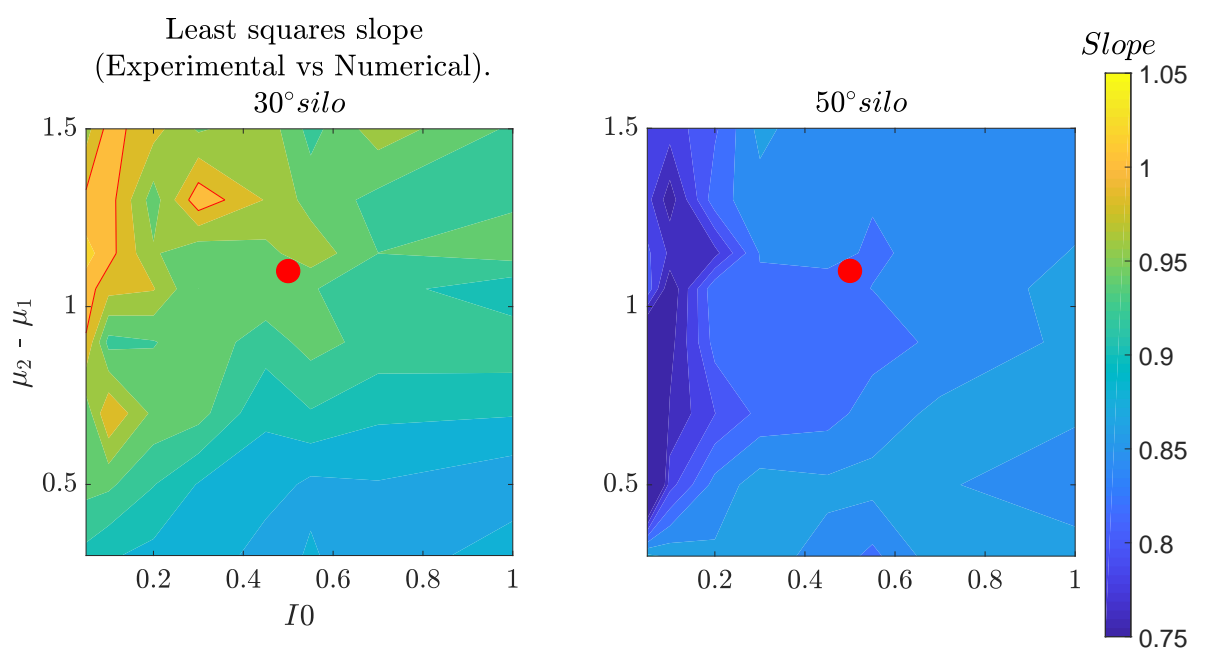

FIG. 14. Sensitivity analysis of the numerical model to parameters $I_{0}$ and $\mu_{2}-\mu_{1}$. The contour plots display the value of the slope found by performing a least-squares linear regression between the experimental and numerical normalised vertical velocity data. The left graph is the analysis for the $30^{\circ}$ silo and the right for the $50^{\circ}$ one. The red dot in the plots indicated the value of the parameters used in the current work, while the fine red line in the left plot is the contour of slope $=1$ (indicating a perfect fit of the numerical to experimental data).

holing flow in a silo is often avoided by smooth-764 ing the silo walls (thus, changing the stress dis-765 tribution in the silo) and/or increasing the size 766 of the silo opening. It is a challenge for simple ${ }_{767}$ incompressible continuum visco-plastic models 68 of granular flow to capture these "finite particle769 size" effects. Further work is needed, includ-770 ing adding the effect of compressibility, to fully 771 capture the observed dynamics in this situation. 772

It is clear that the $\mu(I)$ model performs ad- ${ }_{774}^{773}$ mirably in a silo in the mass and funnel flow ${ }_{775}$ regimes for the parameter values chosen, but $_{776}^{775}$ further model development is needed to fully ${ }_{777}^{776}$ capture the observed phenomena in rat-holing flow, and to accurately predict the flow rate from the silo. Adding in a degree of compressibility into the model and/or accounting for ${ }_{781}^{80}$ granular non-locality and finite size effects may improve flow rate predictions in the silo and ${ }_{783}^{782}$ may help to capture more accurately flowing to ${ }_{784}^{783}$ stagnant phase transitions and potentially the $\mathrm{7}_{785}{ }^{84}$ rat-holing phenomenon [55]. Testing these hy- ${ }_{786}$ potheses is currently being pursued by the au- thors. Additionally, the $\mu(I)$ friction law was discovered using experimental data from relatively low friction spherical particles [56, 57]. It is unclear if the $\mu(I)$ model is the correct friction law to use for natural particles such as the poppy seeds used in this work. Furthermore, particle shape has been shown to be an important factor in the behaviour of general granular systems [58, 59], and silo systems specifically $[60,61]$. Using SEM imaging we found that our poppy seeds were kidney bean shaped, and not spherical. Such an effect could be important to include in a numerical model of granular flow, although the factor does not seem critical, since we obtained very good agreement between experimental and numerical results for the $30^{\circ}$ and $50^{\circ}$ silos. The $\mu(I)$ parameters in the numerical model were our "best guess". The first friction coefficient, $\mu_{1}$, was taken as the angle of repose of the poppy seeds, however, $\mu_{2}$ and $I_{0}$ were chosen to be physically realistic and to try to reduce the ill-posed regions for the $\mu(I)$ model [50]. To check the dependence of model 
results on the $I_{0}$ and $\mu_{2}$ parameters a sensitiv-808 ity analysis was performed. It was found thatsos the accuracy of the model was retained over as10 wide range of parameter values, and that our ${ }_{811}$ choice of $I_{0}$ and $\mu_{2}$ was a good balance of ac-812 curacy for both the $30^{\circ}$ and $50^{\circ}$ silos. To re-813 duce model degrees of freedom these parameters814 should be measured for the specific set of par-815 ticles [62]. In addition to experimentally quan-816 tifying model parameters, the development of 817 realistic numerical boundary conditions should ${ }_{818}$ be a focus. Developing these boundary conditions is a significant future research challenge,, 819 but recent work has made excellent progress to-820 wards this goal $[48,49]$. The observation in the 821 $30^{\circ}$ silo that the flow regime changes from mass ${ }_{822}$ to rat-holing when the boundary condition is823 changed exemplifies the necessity of accurate 24 boundary conditions and may indicate some-825 thing more complex than a simple slip condi-826 tion is needed. Finally, in recent times it has827 been shown that defining an effective granular viscosity using the $\mu(I)$ friction model with an incompressible flow assumption can be mathematically ill-posed depending on the choice of parameters [63]. Adding the effect of compressibility seems to alleviate this issue [50,64]. Although we did not note any issues in our model for our choice of parameters, this fact serves as an additional motivation to transition to a compressible flow model of granular drainage from a silo.

The authors acknowledge the Manawatu Microscopy Imaging Centre (MMIC) at Massey University for producing the SEM images of the poppy seeds in Figure 1. L.F. also acknowledges funding from the Royal Society of New Zealand (contracts RFT-MAU1501-PD and MAU1712). Finally, we acknowledge the help of Maral Mehdizad and Alex Cliff to quantify the mass flow rates in the system.
[1] Bruno Andreotti, Yoël Forterre, and Olivier853 Pouliquen, Granular media: between fluid and 854 solid (Cambridge University Press, 2013). $\quad 855$

[2] Ronald Midgley Nedderman, Statics and kine-856 matics of granular materials (Cambridge Uni-857 versity Press, 2005).

[3] K Kesava Rao, Prabhu R Nott, and S Sundare-859 san, An introduction to granular flow, Vol. $10_{860}$ (Cambridge University Press New York, 2008).861

[4] M Combarros Garcia, HJ Feise, S Strege, and 862 A Kwade, "Segregation in heaps and silos:863 Comparison between experiment, simulation 864 and continuum model," Powder Technology 865 293, 26-36 (2016).

[5] Thomas Weinhart, Carlos Labra, Stefan Lud-867 ing, and Jin Y Ooi, "Influence of coarse-868 graining parameters on the analysis of dem869 simulations of silo flow," Powder technology870 293, 138-148 (2016).

[6] Davide Bertuola, Silvia Volpato, Paolo Canu,872 and Andrea C Santomaso, "Prediction of seg-873 regation in funnel and mass flow discharge," 874 Chemical Engineering Science 150, 16-25875 (2016).

[7] L Staron, P-Y Lagrée, and S Popinet, "Contin-877 uum simulation of the discharge of the granular silo," The European Physical Journal E 37, 5 (2014).

[8] Lydie Staron, P-Y Lagrée, and Stéphane Popinet, "The granular silo as a continuum plastic flow: The hour-glass vs the clepsydra," Physics of Fluids 24, 103301 (2012).

[9] Silvia Volpato, Riccardo Artoni, and Andrea C Santomaso, "Numerical study on the behavior of funnel flow silos with and without inserts through a continuum hydrodynamic approach," Chemical Engineering Research and Design 92, 256-263 (2014).

[10] Yin Wang and Jin Y Ooi, "A study of granular flow in a conical hopper discharge using discrete and continuum approach," Procedia engineering 102, 765-772 (2015).

[11] Sachith Dunatunga and Ken Kamrin, "Continuum modelling and simulation of granular flows through their many phases," Journal of Fluid Mechanics 779, 483-513 (2015).

[12] Luke A Fullard, Clive E Davies, and Graeme C Wake, "Modelling powder mixing in mass flow discharge: A kinematic approach," Advanced Powder Technology 24, 499-506 (2013). 
[13] Luke Fullard, Eric Breard, Clive Davies,931 Pierre-Yves Lagrée, Stéphane Popinet, and932 Gert Lube, "Testing the $\mu$ (i) granular rheology93з against experimental silo data," in EPJ Web of $f_{934}$ Conferences, Vol. 140 (EDP Sciences, 2017) p.935 11002.

[14] Y Zhou, P-Y Lagrée, S Popinet, P Ruyer, and937 Pascale Aussillous, "Experiments on, and dis-938 crete and continuum simulations of, the dis-939 charge of granular media from silos with a lat-940 eral orifice," Journal of Fluid Mechanics 829,941 459-485 (2017).

15] LA Fullard, CE Davies, AC Neather, ECPors Breard, AJR Godfrey, and G Lube, "Test-944 ing steady and transient velocity scalings in a945 silo," Advanced Powder Technology 29, 310-946 318 (2018).

[16] LA Fullard, CE Davies, G Lube, AC Neather,948 ECP Breard, and BJ Shepherd, "The tran-949 sient dynamics of dilation waves in granular950 phase transitions during silo discharge," Gran-951 ular Matter 19, 6 (2017).

952

[17] Jaehyuk Choi, Arshad Kudrolli, and Martin Z953 Bazant, "Velocity profile of granular flows in-954 side silos and hoppers," Journal of Physics:955 Condensed Matter 17, S2533 (2005)

[18] A Janda, Iker Zuriguel, A Garcimartín, Luis A957 Pugnaloni, and Diego Maza, "Jamming and958 critical outlet size in the discharge of a two-959 dimensional silo," EPL (Europhysics Letters)960 84, $44002(2008)$.

[19] I Sielamowicz, S Blonski, and TA Kowalewski,962 "Optical technique dpiv in measurements of 963 granular material flows, part 1 of 3plane hop-964 pers," Chemical Engineering Science 60, 589-965 598 (2005).

[20] K Endo, K Anki Reddy, and H Katsuragi,967 "Obstacle-shape effect in a two-dimensional968 granular silo flow field," Physical Review Flu-969 ids 2, 094302 (2017).

970

[21] Selam Waktola, Andre Bieberle, Frank971 Barthel, Martina Bieberle, Uwe Hampel,972 Krzysztof Grudzień, and Laurent Babout,973 "A new data-processing approach to study974 particle motion using ultrafast x-ray tomogra-975 phy scanner: case study of gravitational mass976 flow," Experiments in Fluids 59,69 (2018). 9977

[22] Krzysztof Grudzien, Maciej Niedostatkiewicz,978 Jerome Adrien, Eric Maire, and Laurent979 Babout, "Analysis of the bulk solid flow during 980 gravitational silo emptying using x-ray and ect981 tomography," Powder technology 224, 196-208982 (2012).
[23] H-Y Xie and K Shinohara, "Measurement of solids velocity in a conical hopper by mass tracer particles," Chemical engineering science 54, 455-459 (1999).

[24] PA Langston, MS Nikitidis, U Tüzün, DM Heyes, and NM Spyrou, "Microstructural simulation and imaging of granular flows in two-and three-dimensional hoppers," Powder Technology 94, 59-72 (1997).

[25] MS Nikitidis, U Tüzün, and NM Spyrou, "Determination of phase velocities in multi-phase flows in hoppers using dual photon gamma-ray tomography," Chemical Engineering Communications 175, 3-24 (1999).

[26] Michail S Nikitidis, Ugur Tüzün, and Nicholas M Spyrou, "Tomographic measurements of granular flows in gases and in liquids," KONA Powder and Particle Journal 12, 53-67 (1994).

[27] Ralf Stannarius, "Magnetic resonance imaging of granular materials," Review of Scientific Instruments 88, 051806 (2017).

[28] Alexander Penn, Takuya Tsuji, David O Brunner, Christopher M Boyce, Klaas P Pruessmann, and Christoph R Müller, "Real-time probing of granular dynamics with magnetic resonance," Science Advances 3, e1701879 (2017).

[29] Christopher M Boyce, Alexander Penn, Klaas P Prüssmann, and Christoph Rüdiger Müller, "Magnetic resonance imaging of gassolid fluidization with liquid bridging," AIChE Journal (2017).

[30] CM Boyce, NP Rice, A Ozel, JF Davidson, Andrew John Sederman, Lynn Faith Gladden, S Sundaresan, John Stephen Dennis, and DJ Holland, "Magnetic resonance characterization of coupled gas and particle dynamics in a bubbling fluidized bed," Physical Review Fluids 1, 074201 (2016).

[31] Hilary T Fabich, Andrew J Sederman, and Daniel J Holland, "Development of ultrafast ute imaging for granular systems," Journal of Magnetic Resonance 273, 113-123 (2016).

[32] CM Boyce, A Ozel, NP Rice, GJ Rubinstein, DJ Holland, and S Sundaresan, "Effective particle diameters for simulating fluidization of non-spherical particles: Cfd-dem models vs. mri measurements," AIChE Journal 63, 25552568 (2017)

[33] Hilary T Fabich, Andrew J Sederman, and Daniel J Holland, "Study of bubble dynamics in gas-solid fluidized beds using ultrashort echo 
time (ute) magnetic resonance imaging (mri),"1037 Chemical Engineering Science 172, 476-486038 (2017).

1039

[34] HT Fabich, TI Brox, D Clarke, JD Seymour 3040 SL Codd, P Galvosas, J Brown, AJ Sedermanı041 and DJ Holland, "Measurements of the velocityro42 distribution for granular flow in a couette cell,"1043 Physical Review E 98, 062901 (2018).

1044

[35] Toshihiro Kawaguchi, "Mri measurement of 045 granular flows and fluid-particle flows," Ad+046 vanced Powder Technology 21, 235-241 (2010)1047

[36] Michael Gentzler and Gabriel I Tardos, "Meat048 surement of velocity and density profiles ino49 discharging conical hoppers by nmr imaging,"1050 Chemical Engineering Science 64, 4463-4469051 (2009).

[37] ZH Gu, PC Arnold, and AG McLean, "Preł053 diction of the flowrate of bulk solids from mass054 flow bins with conical hoppers," Powder tech 4055 nology 72, 157-166 (1992).

[38] Pierre Jop, Yoël Forterre, and Olivierno57 Pouliquen, "A constitutive law for dense gran 4058 ular flows," Nature 441, 727 (2006).

[39] P-Y Lagrée, Lydie Staron, and Stéphane060 Popinet, "The granular column collapse as061 a continuum: validity of a two-dimensionalo62 navier-stokes model with a $\mu$ (i)-rheology,"1063 Journal of Fluid Mechanics 686, 378-408064 (2011).

1065

[40] Przemyslaw B Kowalczuk and Jan Drzymalało66 "Physical meaning of the sauter mean diame 067 ter of spherical particulate matter," Particulate068 Science and Technology 34, 645-647 (2016). 1069

[41] Kiwing To, "Jamming transition in twot070 dimensional hoppers and silos," Physical Reł071 view E 71, 060301 (2005).

[42] Nan Gui, Xingtuan Yang, Jiyuan Tu, and073 Shengyao Jiang, "Effects of rocking frequencyior4 and amplitude on particle discharge in rocking 075 bed: A dem study," Powder technology 292 1076 31-45 (2016).

1077

[43] Arnold McLean, "The use of standpipes foro78 increasing limiting gravitational flowrate fromo79 mass flow bins," KONA Powder and Particleoso Journal 11, 139-145 (1993).

1081

[44] C Mankoc, A Janda, Roberto Arevalo, JM Pasto82 tor, Iker Zuriguel, A Garcimartín, and Diego08з Maza, "The flow rate of granular materials084 through an orifice," Granular Matter 9, 407-1085 414 (2007).

[45] W Ar Beverloo, Hendrik Antonie Leniger, and 087 J Van de Velde, "The flow of granular solidso8s through orifices," Chemical engineering scienceosg
15, 260-269 (1961).

[46] Paul T. Callaghan, Translational Dynamics and Magnetic Resonance, 1st ed. (Oxford University Press, Oxford, 2011).

[47] Stéphane Popinet, "Gerris: a tree-based adaptive solver for the incompressible euler equations in complex geometries," Journal of Computational Physics 190, 572-600 (2003).

[48] Riccardo Artoni, Andrea C Santomaso, Massimiliano Go, and Paolo Canu, "Scaling laws for the slip velocity in dense granular flows," Physical review letters 108, 238002 (2012).

[49] Riccardo Artoni and Andrea Santomaso, "Effective wall slip in chutes and channels: experiments and discrete element simulations," Granular Matter 16, 377-382 (2014).

[50] Thomas Barker, DG Schaeffer, Michael Shearer, and JMNT Gray, "Well-posed continuum equations for granular flow with compressibility and $\mu$ (i)-rheology," Proc. R. Soc. A 473, 20160846 (2017).

[51] Luke Fullard and Clive Davies, "Minimising the spread of residence-time distribution for flat and heaped powders in a wedge-shaped planar hopper," Particuology 30, 102-110 (2017).

[52] Kenneth E Fickie, Reza Mehrabi, and Roy Jackson, "Density variations in a granular material flowing from a wedge-shaped hopper," AIChE journal 35, 853-855 (1989).

[53] RL Brown, "Minimum energy theorem for flow of dry granules through apertures," Nature 191, 458 (1961).

[54] Mebirika Benyamine, Pascale Aussillous, and Blanche Dalloz-Dubrujeaud, "Discharge flow of a granular media from a silo: effect of the packing fraction and of the hopper angle," in EPJ Web of Conferences, Vol. 140 (EDP Sciences, 2017) p. 03043.

[55] David L Henann and Ken Kamrin, "A predictive, size-dependent continuum model for dense granular flows," Proceedings of the National Academy of Sciences 110, 6730-6735 (2013).

[56] GDR MiDi, "On dense granular flows," The European Physical Journal E 14, 341-365 (2004).

[57] Pierre Jop, Yoël Forterre, and Olivier Pouliquen, "Crucial role of sidewalls in granular surface flows: consequences for the rheology," Journal of Fluid Mechanics 541, 167-192 (2005).

[58] Emilien Azéma, Nicolas Estrada, Itthichai Preechawuttipong, Jean-Yves Delenne, and Farhang Radjai, "Systematic description of the 
1090 effect of particle shape on the strength proper ${ }+06$ ties of granular media," in EPJ Web of Confer ${ }_{1107}$ ences, Vol. 140 (EDP Sciences, 2017) p. 060261108

[59] Shiwei Zhao and Xiaowen Zhou, "Effects of 109 particle asphericity on the macro-and micro 1110 mechanical behaviors of granular assemblies," 1111 Granular Matter 19, 38 (2017).

1112

60] Tamás Börzsönyi, Ellák Somfai, Balázs Szabół113 Sandra Wegner, Ahmed Ashour, and Ralf 114 Stannarius, "Elongated grains in a hopper," in115 EPJ Web of Conferences, Vol. 140 (EDP Sci+116 ences, 2017) p. 06017.

[61] Ezequiel Goldberg, C Manuel Carlevaro, and118 Luis A Pugnaloni, "Effect of grain shape on 119 the jamming of two-dimensional silos," in EP $d_{120}$ Web of Conferences, Vol. 140 (EDP Sciences,
2017) p. 06009

[62] Abdoulaye Fall, Guillaume Ovarlez, David Hautemayou, Cédric Mézière, J-N Roux, and François Chevoir, "Dry granular flows: Rheological measurements of the $\mu$ (i)-rheology," Journal of rheology 59, 1065-1080 (2015).

[63] T Barker, DG Schaeffer, P Bohorquez, and JMNT Gray, "Well-posed and ill-posed behaviour of the $\mu(i)$-rheology for granular flow," Journal of Fluid Mechanics 779, 794-818 (2015).

[64] Joris Heyman, R Delannay, H Tabuteau, and A Valance, "Compressibility regularizes the $\mu$ (i)-rheology for dense granular flows," Journal of Fluid Mechanics 830, 553-568 (2017). 